\title{
Imagem corporal e comportamento alimentar em estudantes universitárias
}

\section{Body image and food behavior in university students}

\author{
Carlos Eduardo Vilela Gaudioso* \\ José Carlos SouzA** \\ Luis Alberto Magna*** \\ Heloísa Nunes BotelHo****
}

\begin{abstract}
Resumo
Este estudo avaliou a imagem corporal e as atitudes alimentares de uma amostra de estudantes universitárias de uma faculdade do Mato Grosso do Sul, Brasil. Método: quantitativo, exploratório, descritivo e de corte transversal. Participaram da pesquisa 106 alunas. Foram aplicados três instrumentos, sendo um questionário sócio-demográfico; para avaliar a insatisfação com a imagem corporal foi utilizado o Questionário de Imagem Corporal (Body Shape Questionnaire-BSQ) e para a identificação da presença de distúrbios de atitudes alimentares foi utilizado o Teste de Atitudes Alimentares (Eating Attitude Test-EAT-26). Resultados indicaram, pelo EAT26, que $15,2 \%$ das alunas
\end{abstract}

* Psicólogo, Mestre em Psicologia da Saúde, Doutorando em Ciências Médicas da Universidade Estadual de Campinas (UNICAMP), Professor da Universidade Federal de Mato Grosso do Sul (UFMS), campus Coxim.

* Psiquiatra, Doutor em Saúde Mental - Unicamp, Professor do curso de medicina da Universidade Estadual de Mato Grosso do Sul (UEMS), Campo Grande

*** Médico Geneticista, Livre-docente em Genética Médica, Professor titular do Departamento de Genética Médica da UNICAMP, Bacharel em Direito e advogado

**** Acadêmica do curso de Enfermagem da Universidade Federal de Mato Grosso do Sul (UFMS), campus Coxim. 
tinham escore sugestivo de distúrbio alimentar (acima de 21) e $84,8 \%$ escore igual ou inferior a 21, portanto indicativo de ausência de distúrbio alimentar. Tanto a idade quanto o IMC e a frequência semanal de atividade física são semelhantes nos grupos identificados pelo EAT26 como sem distúrbio ou com provável distúrbio alimentar. As médias para a idade em cada grupo foram 24,86 e 25,44 anos; IMC médio de 21,78 e 22,77; e média de 3,83 e 3,33 de vezes/semana de prática de atividade física. Em relação ao BSQ, seu resultado foi conclusivo para 95 alunas, as quais se distribuíram em $74,7 \%$ sem transtorno, $11,6 \%$ com transtorno leve, $8,4 \%$ com transtorno moderado e $5,3 \%$ com transtorno grave.

Palavras-chave: Imagem corporal; Comportamento alimentar; Estudantes.

\section{Abstract}

This study evaluated the body image and eating attitudes of a sample of university students from a college Mato Grosso do Sul, MS, Brazil. Method: quantitative, exploratory, descriptive and cross-sectional. 106 students participated in the study. Three instruments were applied, being a socio-demographic questionnaire; To evaluate body image dissatisfaction, the Body Shape Questionnaire (BSQ) was used and the Eating Attitude Test (EAT-26) was used to identify the presence of eating disorders. Results: $15.2 \%$ of the students with a suggestive eating disorder score (above 21 ) and $84.8 \%$ with a score equal to or lower than 21 were presented, by the application of the EAT instrument26, therefore indicative of absence of eating disorder. Both age, BMI and weekly physical activity frequency are similar in the groups identified by the EAT26 as without disturbance or with probable eating disorder. The averages for age in each group were 24.86 and 25.44 years; Mean BMI of 21.78 and 22.77; And a mean of 3.83 and 3.33 times / week of physical activity. Regarding BSQ, the results were $95 \%$ for the students, who were distributed in $74.7 \%$ without disorder, $11.6 \%$ with mild disorder, $8.4 \%$ with moderate disorder and $5.3 \%$ with severe disorder.

Keywords: Body image. Eating behavior. Students.

\section{Introdução}

O corpo é o meio de contato primário do individuo com o ambiente que o cerca. Através do corpo que as primeiras relações 
são estabelecidas; tanto que Kofes (1985) afirma que o corpo é a expressão da cultura, portanto, cada cultura vai se expressar por meio de diferentes corpos. Existe uma preocupação com a perfeição estética do corpo, um verdadeiro paradoxo do que é desejável e daquilo que é aceitável socialmente, esta, por sua vez determina padrões que interferem significativamente nas convicções e apelam para a materialidade capitalista, cultuada pela indústria da moda provocando estímulos ao consumo de cosméticos, de cirurgias plásticas, de carreiras de artistas, manequins e atletas, violando as concepções de saúde, ocasionando a inquietação de diversas áreas interessadas na qualidade de vida do ser humano.

O corpo magro, venerado pela maioria das adolescentes, tem tido a primazia do cuidado com o peso, no intuito de buscar um biótipo aceitável para a época atual. Cabe ressaltar que a descrição dos transtornos alimentares iniciou-se em um momento em que os padrões estéticos aceitáveis eram os arredondados e com curvas marcantes. Isto foi ressaltado pelas obras dos Renascentistas, admiradores das formas boleadas que retratavam os corpos atrativos da época. À medida que suas obras conquistavam outras regiões do mundo a indústria cosmética, alimentícia e de beleza tentava padronizar o corpo esbelto e magro, aproveitando campanhas na promoção de saúde e incentivo a magreza, acreditando que está seria a forma saudável de apresentação corporal, conforme Almeida (2005).

Como explicou Ballone (2003), pacientes anoréxicos e bulímicos apresentam alterações emocionais, antes mesmo da manifestação da doença alimentar, tais como: insatisfações com alguma parte do corpo, descontentamento com o peso, características da alteração na percepção corporal. Afirma ainda esse mesmo autor, que esses pacientes possuem o hábito de praticar dietas até mesmo quando há proporcionalidade entre peso e estatura ou, mesmo que obtenham resultados de diminuição de peso, continuam a praticar dietas. O autor afirma que "dismorfia corporal" é a primeira experiência que antecede o transtorno alimentar, e caracteriza-se por ser uma obsessão relacionada com alguma parte do corpo, que gera preocupação exagerada, e causa sofrimento e prejuízo no seu funcionamento social e ocupacional. 
A imagem corporal e atitudes alimentares são construções culturais e biológicas, adotam-se símbolos significativos para a sobrevivência social. Existimos e nos distinguimos dos animais pela forma como compreendemos esses símbolos. A imagem corporal segue a premissa do imaginário e nele é representada esta preocupação inerente a pessoas que lidam com o corpo e com a criatividade. Esses processos vão existir e serão proeminentes para a vida diante dos anseios e perspectivas dentro do mundo globalizado influenciado pela mídia.

Este trabalho apresenta os resultados da pesquisa com método de natureza quantitativa, exploratória, descritiva e de corte transversal realizada em um município do interior do estado do Mato Grosso do Sul, realizada em 2009. Assim, os objetivos desse estudo foram:

- objetivo geral : relacionar imagem corporal com comportamento alimentar de uma amostra de estudantes universitárias de uma faculdade do Mato Grosso do Sul.

Como objetivos específicos: - Caracterizar o perfil sociodemográfico das estudantes; Comparar os dados sociodemográficos da amostra em relação à presença ou não de transtorno da imagem corporal; Comparar os dados sociodemográficos da amostra em relação às atitudes alimentares

\section{Método}

O presente estudo trata-se de uma pesquisa de natureza quantitativa, exploratória, descritiva e de corte transversal realizada em um município do interior do estado do Mato Grosso do Sul, realizada em 2009.

Amostra - participaram da pesquisa 122 estudantes, do sexo feminino, cursando do $1^{\mathrm{o}}$ ao $8^{\mathrm{o}}$ semestres de todos os cursos superiores da Faculdade MAGSUL, o que corresponde a aproximadamente $38 \%$ da população total de 316 alunas regularmente matriculadas em 2007.

Critérios de inclusão - foram incluídas as alunas devidamente matriculadas nos cursos de graduação no $2^{\underline{0}}$ semestre do ano de 2007, que estavam em sala de aula no momento da coleta de dados.

Critérios de exclusão - foram excluídas as estudantes que se encontravam em licença médica ou aquelas que faltaram às aulas nos dias em que se deu a coleta de dados. 
Local - a pesquisa foi realizada na Faculdade de Educação Ciências e Letras de Ponta Porã (FECLEPP), em Mato Grosso do Sul, também chamada de Faculdade MAGSUL. Com um número total de 511 alunos, sendo 316 do sexo feminino e 195 do sexo masculino, de diversas cidades pertencentes ao Conesul do Estado. São cidades pequenas com o índice populacional de aproximadamente 10.000 (dez mil) habitantes, que migram para Ponta Porã para cursarem a graduação desejada. A região oferece a multiculturalidade pois, a localização geográfica divide dois países, Brasil e Paraguai pelas cidades de Ponta Porã e Pedro Juan Caballero, a cidade brasileira segue o padrão da região de agropecuária com a economia centrada neste aspecto, já a cidade do País vizinho, centra sua economia no turismo de compras pela variedade de produtos importados com preços atrativos aos brasileiros. Uma região com a população pacata e na sua maioria procedente do meio rural.

\section{Instrumentos}

- Questionário Dados pessoais - elaborado especialmente para o presente estudo, o quetionário levantou as variáveis: idade, peso e altura (convertidos a IMC para as finalidades do estudo). Levantou antecedentes como: se já teve depressão, se praticava atividades físicas (freqüência semanal e tipo de atividades).

- Body Shape Questionnaire - BSQ. Para avaliar a satisfação com a imagem corporal, foi utilizado o Questionário de Imagem Corporal (Body Shape Questionnaire - BSQ) - versão para mulheres, desenvolvido por Cooper et al. (1987), traduzido por Cordás e Neves (1999) e validado para uma população de universitários brasileiros por Di Pietro (2002). O BSQ é um instrumento de domínio público que mede o grau de preocupação com a forma do corpo, a autodepreciação devido à aparência física e a sensação de estar obeso. Consta de 34 questões com seis opções de resposta: 1- Nunca; 2- Raramente; 3- Às Vezes; 4- Freqüentemente; 5- Muito Freqüentemente; 6- Sempre. A classificação dos resultados é dividida em quatro níveis de transtorno da auto-imagem corporal. A pontuação abaixo de 80 indica Ausência de distorção; pontuação entre 80 e 110 indica distorção Leve; entre 110 e 140 indica distorção Moderada e pontuação igual ou acima de 140 - distorção grave da auto- imagem corporal. 
- Eating Attitude Test - EAT-26 - Para a identificação da presença de distúrbios de atitudes alimentares foi utilizado o Teste de Atitudes Alimentares (Eating Attitude Test - EAT-26), que é um dos instrumentos mais utilizados atualmente, tendo sido traduzido em quase uma dezena de línguas estrangeiras (MINTZ; O'HALLORAN, 2000). É de domínio público, sendo um teste psicométrico utilizado em estudos sobre anorexia nervosa com o objetivo de medir sintomas da síndrome, favorecendo o diagnóstico e o tratamento precoces. Esse instrumento foi desenvolvido por Garner e Garfinkel (1979), que utilizaram uma população feminina do Canadá. A versão original continha 40 itens Posteriormente Garner et al. (1982) desenvolveram a versão abreviada com 26 questões, mantendo as correlações psicométricas, entre pacientes e participantes normais, da versão original. O ponto de corte estabelecido para o EAT-26 foi 21 pontos. As 26 questões estão assim divididas nas três escalas ou fatores obtidos a partir de análise fatorial, cujos conceitos e números são: escala da dieta, reflete uma recusa patológica a altos teores calóricos e grande preocupação com a forma física. A escala de bulimia, refere-se à ingestões compulsivas de alimentos, seguidas de vômitos e outros comportamentos evitativos de ganho de peso. Por último, a escala de controle oral, refere-se ao auto controle em relação aos alimentos e reconhece forças sociais que estimulam a ingestão alimentar. Cada pergunta e dividida em três escalas do tipo Likert e apresenta seis opções de resposta, conferindo-se pontos de 0 a 3, dependendo da escolha (Sempre = 3 ponto; Muito Freqüentemente $=2$ ponto; Freqüentemente $=1$ ponto; Às Vezes $=0$ ponto; Raramente $=0$ ponto; Nunca $=0$ ponto). A única questão que apresenta pontos em ordem invertida é a de número 25, sendo que para respostas mais sintomáticas como o sempre, muito freqüentemente e freqüentemente, não são dados pontos, e para as alternativas às vezes, raramente, e nunca, são conferidos 1, 2 e 3 pontos, respectivamente. Um resultado maior que 21 pontos indica um teste positivo e o participante poderá ser incluído em grupo de risco para desenvolver transtornos alimentares (GARNER; GARFINKEL, OLMSTEAD, 1979, GARNER; GARFINKEL; OLMSTEAD, 1983).

A nálise de dados - os métodos estatísticos empregados foram: métodos de estatística descritiva, gráfica e aritmética; comparação 
de proporções, no caso das variáveis de atributo ou categóricas, pelo qui-quadrado ou teste exato de Fisher, conforme a indicação de cada um deles; comparação de médias, no caso das variáveis quantitativas, pelo método $t$ de Student para amostras independentes e pelo modelo aleatório de análise da variância (Oneway ANOVA) com comparação posterior pelo método Least Square Difference (LSD). Para a comparação de proporções foi utilizado o método do qui-quadrado. Utilizou-se também a correlação simples de Pearson e o modelo linear de regressão múltipla escalonada. Em todos os casos a declaração de significância se deu no nível de $5 \%(p<0,05)$.

\section{Resultados e Discussão}

Pelos resultados obtidos (conforme Tabela 1) pode-se observar uma população saudável em comparação a outros estudos já realizados, como de Nakamura et al. (1999) em que foi utilizado EAT-26 em 3.032 estudantes de três escolas privadas para avaliar problemas alimentares e seus dados mostraram $5,4 \%$ dos sujeitos apresentaram alto escore. Também Ribeiro (1999) levantou a possibilidade da alta prevalência em jovens com hábitos dietéticos que podiam indicar riscos para o desenvolvimento de anorexia nervosa e bulimia; além de sugerirem aspectos cognitivos e comportamentais semelhantes aos doentes clinicamente diagnosticados. Também é importante salientar que no presente estudo observou-se que a escala de dieta do EAT-26 e os escores do BSQ não apresentaram relevância para o surgimento de transtornos futuros.

TABELA 1 - Categoria BSQ e categoria EAT-26

\begin{tabular}{c|c|c|c|c|c|c}
\hline \multirow{2}{*}{ BSQ } & \multicolumn{4}{|c|}{ EAT-26 } & \multicolumn{2}{c}{ Total } \\
\cline { 2 - 6 } & \multicolumn{2}{|c|}{ Sem distúrbio } & \multicolumn{2}{c}{ Com distúrbio } & \multicolumn{2}{c}{} \\
\cline { 2 - 6 } & $\mathbf{n}$ & $\mathbf{\%}$ & $\mathbf{n}$ & $\mathbf{\%}$ & $\mathbf{n}$ & $\mathbf{\%}$ \\
\hline Sem transtorno & 64 & 90,1 & 7 & 9,9 & 71 & 100,0 \\
Transtorno leve & 8 & 72,7 & 3 & 27,3 & 11 & 100,0 \\
Transtorno & 6 & 75,0 & 2 & 25,0 & 8 & 100,0 \\
$\begin{array}{c}\text { moderado } \\
\text { Transtorno grave }\end{array}$ & 2 & 40,0 & 3 & 60,0 & 5 & 100,0 \\
\hline Total & $\mathbf{8 0}$ & $\mathbf{8 4 , 2}$ & $\mathbf{1 5}$ & $\mathbf{1 5 , 8}$ & $\mathbf{9 5}$ & $\mathbf{1 0 0 , 0}$ \\
\hline
\end{tabular}


Com relação aos resultados referentes a prática ou não de atividades físicas, observou-se que esses não mostraram semelhança na proporção da ocorrência do provável distúrbio alimentar ou mesmo distorção da imagem corporal, o que contradiz outros resultados da literatura. Os resultados sugeriram que a inatividade física era resultante do estilo de vida adotado. Estilo esse voltado para atividades de trabalho, bem como e de lazer caracterizados por atividades inertes que favorecem o aumento da prevalência de obesidade em todas as faixas etárias. É interessante observar que o desejo de ter ou permanecer com o corpo magro não encontra correspondência com o perfil de prática de atividades físicas do grupo e contraria, ainda, o discurso científico da nutrição, que preconiza a atividade física como elemento relevante na promoção da saúde e do corpo saudável. Assim, o presente estudo mostrou que somente $15,2 \%$ das estudantes tinham algum escore sugestivo de distúrbio alimentar (acima de 21).

Confrontando esses resultados com a literatura, é possível observar um estudo realizado por Pokrajac-Bulian; Zivcic-Becirevicacute (2005) com universitários croatas em que se utilizou o BSQ; os resultados mostram que a insatisfação corporal tinha um papel significativo na auto-estima das meninas; e quando se comparou as croatas com, com estudantes do sexo feminino do Reino Unido e dos Estados Unidos, verificou-se que seus escores eram significativamente mais baixos. Comparando os resultados com alguns achados disponíveis na literatura nacional, observam-se que a pontuação média no BSQ das estudantes universitárias no presente estudo foi $81,2( \pm 33,6)$, ligeiramente mais alta que a encontrada em estudo entre atletas realizado por Oliveira et al. (2003) no Rio de Janeiro, que foi $77,8( \pm 28,7)$.

Outros dados interessantes foram apresentados por Almeida et al. (2005) em que encontrou $74,7 \%$ de sua amostra sem transtorno, $11,6 \%$ com transtorno leve para percepção de imagem corporal de mulheres atendidas em diferentes serviços ambulatoriais de saúde de Ribeirão Preto, SP. Os autores observaram uma concentração de escolhas próprias de não-obesidade nas silhuetas representativas de mulheres caracterizando a exigência de corpos magros como sinônimo de normalidade. 
Considerando, porém que o número de sujeitos com algum tipo de transtorno segundo as categorias de BSQ é pequeno. Isto porque se separarmos os sujeitos, segundo as categorias de BSQ, como sem transtorno ou tendo algum transtorno (leve, moderado ou grave), o que aumenta a precisão do teste estatístico, chega-se à conclusão de que mais provavelmente exista uma associação entre transtorno de BSQ (de qualquer grau) e distúrbio alimentar segundo a pontuação EAT-26 ( $p=0,006)$. Podemos verificar estes dados na tabela 2 . Um estudo longitudinal entre meninas norueguesas apontou que a imagem corporal é fator preditor para a prática de dietas, tendo relação direta com o aumento da idade (FRIESTAD; RISE, 2004). E a prática de dietas freqüentes é fator de risco para o desenvolvimento de transtornos do comportamento alimentar, sobretudo de quadros de anorexia nervosa (MORGAN; VECCHIATTI; NEGRÃO, 2002).

Das variáveis dependentes consideradas (idade, IMC, prática de atividade física, freqüência de atividade física na semana, depressão anterior e categorias de BSQ), somente a última está relacionada com provável distúrbio alimentar detectado pela aplicação das categorias do instrumento EAT-26 ( $\mathrm{p}=0,013)$, o que se dá, mais provavelmente, pelo fato de que aumenta a proporção de sujeitos com provável distúrbio alimentar entre aqueles que manifestam algum grau de transtorno da imagem corporal $(p=0,006)$. Pokrajac-Bulian e Ambrosi-Randic (2007) estudaram 471 mulheres croatas de 14 a 23 anos de idade, através do BSQ e detectaram graus severos de insatisfação corporal que estavam significativamente relacionados com a internalização e consciência do ideal de magreza.

TABELA II - Escala de Dieta - EAT-26 e variáveis; depressão, atividade física e categorias de BSQ.

\begin{tabular}{|c|c|c|c|c|c|c|}
\hline \multirow{2}{*}{$\begin{array}{l}\text { Variável } \\
\text { independente }\end{array}$} & \multirow{2}{*}{ Descrição } & \multicolumn{4}{|c|}{ Escala de Dieta } & \multirow[b]{2}{*}{$\mathrm{p}$} \\
\hline & & $\mathbf{N}$ & média & $\mathrm{Dp}$ & epm & \\
\hline \multirow[t]{3}{*}{ Teve depressão } & Não & 79 & 15,04 & 8,53 & 0,96 & \multirow{3}{*}{0,113} \\
\hline & Sim & 32 & 18,91 & 12,38 & 2,19 & \\
\hline & Total & 111 & 16,15 & 9,90 & 0,94 & \\
\hline \multirow[t]{2}{*}{ Atividade física } & $\begin{array}{l}\text { Não } \\
\text { Sim }\end{array}$ & 61 & 16,33 & 10,17 & 1,30 & \multirow[t]{2}{*}{0,766} \\
\hline & Total & 112 & 16,07 & 9.89 & 0,93 & \\
\hline
\end{tabular}




\begin{tabular}{lllllll}
\hline Categorias de BSQ & $\begin{array}{l}\text { Sem } \\
\text { transtorno }\end{array}$ & 76 & 12,33 & 7,21 & 0,83 & \\
& $\begin{array}{l}\text { Transtorno } \\
\text { leve }\end{array}$ & 11 & 24,82 & 5,15 & 1,55 & 0,000 \\
& $\begin{array}{l}\text { Transtorno } \\
\text { moderado }\end{array}$ & 8 & 25,38 & 6,70 & 2,37 & \\
$\begin{array}{l}\text { Transtorno } \\
\text { grave }\end{array}$ & 5 & 33,40 & 6,84 & 3,06 \\
\hline & Total & $\mathbf{1 0 0}$ & $\mathbf{1 5 , 8 0}$ & $\mathbf{9 , 4 1}$ & $\mathbf{0 , 9 4}$ \\
\hline
\end{tabular}

Rodríguez et al. (2001) avaliaram 403 adolescentes, 191 espanholas e 212 latino americanas e detectaram que as últimas têm piores escores no BSQ, portanto mais insatisfação com a imagem corporal, devido, segundo o estudo, à maior influência dos fatores socioculturais entre as latino americanas.

Alves et al. (2008) estudaram 1.148 adolescentes brasileiras de Florianópolis, SC, detectaram 15,6\% delas com EAT + e 18,8\% com insatisfação da imagem corporal, o EAT + foi associado com idade de 10 a 13 anos $(p=0,046)$ sobrepeso e obesidade $(p<0,001)$, insatisfação com a imagem corporal $(\mathrm{p}<0,001)$.

Esses dados confirmam que a percepção do peso corporal se sobrepõe ao IMC, ou seja, a forma como a pessoa se percebe é mais decisiva do que a massa corporal em si, podendo influenciar alterações importantes do comportamento alimentar. Esse fato evidencia a necessidade de explorar o tema em diferentes segmentos populacionais, de modo a conhecer a magnitude do fenômeno e delinear estratégias voltadas ao problema (NUNES et al., 2001).

Quando há relação dos processos internos das pessoas relacionados com o seu comportamento. $\mathrm{O}$ comportamento se modificara conforme os estímulos influências, pessoas afetadas psicologicamente e emocionalmente, o sentimento de tristeza, irritabilidade e agressividade pode ser um quadro do início de patologias. A relação apropriada para o presente estudo configura um dado comportamental e simbólico criado pelo ser humano, os estudos de Strubbe e Gorissen sobre saciedade apontados nesta pesquisa e de Gietzen sobre dietas apontam a necessidade de observar e avaliar o indivi- 
duo como um todo, as perspectivas deste ser que agoniza algo além de sua consciência, distingue suas vontades psíquicas; o corpo real e o corpo ideal são premissas que se estabelecem na criação do ser, ou na iniciação da vida partindo dos processos mais primitivos de reconhecimento do próprio eu.

\section{Conclusão}

A autopercepção da imagem corporal e, principalmente, a imagem corporal idealizada pelas universitárias informantes deste estudo ajustam-se ao padrão preconizado pelo contexto sociocultural em que vivemos, no qual sobressaem o que certos autores denominam lipofobia e império da magreza. Em se tratando de graduandas de cursos variados, o impacto desse achado é ainda mais relevante, pois se profissionais no campo da saúde e da educação, apesar de uma compreesão nesses domínios, desejam atender a essas normas, evidencia-se a importância do tema como questão sócio-sanitária. Além disso, a condição de cuidadores adoecidos dificulta a construção de um olhar diferenciado para com o outro no momento em que tiverem que lidar com algum caso de transtorno alimentar, obscurecendo a distorção da auto-imagem como preditora para o desenvolvimento de transtornos alimentares.

O desconhecimento do tema por parte dos profissionais das áreas afins gerado pelo despreparo acadêmico associado ao caráter secreto das práticas torna mais difícil a sua detecção. Portanto, a multidimensionalidade dos transtornos do comportamento alimentar, que inclui a percepção do próprio corpo, deve ser discutida com esses profissionais para que entendam a influência da sociedade de consumo e o processo de construção dos ideais de beleza nos processos de subjetivação em curso e possam conduzir um manejo clínico no qual se considerem os limites nos quais as alterações da imagem corporal e do comportamento alimentar trará impacto relevante à saúde das pessoas.

As atitudes alimentares das universitárias são basicamente influenciadas pela imagem corporal que as mesmas têm de si próprias, havendo nítida tendência de ocorrência de maiores escores nas três escalas alimentares consideradas (dieta, bulimia e controle oral) com o aumento da pontuação de BSQ, estabelecendo significativa 
associação entre as atitudes alimentares em cada uma das escalas e as categorias de transtorno de imagem corporal.

A idade, o IMC, a ocorrência de depressão prévia e a prática de atividade física, por outro lado, não são variáveis que influenciam significativamente as atitudes alimentares da população de universitárias representada pela amostra estudada.

O transtorno de imagem corporal, segundo a escala de BSQ, por sua vez, mostra uma associação positiva com o IMC, aumentando o grau de transtorno da imagem corporal com o aumento do IMC. A idade e a prática de atividade física não mostraram associação com o grau de transtorno de imagem corporal, mas se pode perceber tendência de maior proporção de ocorrência de depressão prévia no grupo com transtorno grave da imagem corporal, o que não pôde ser demonstrada na presente amostra pelo pequeno número de casos.

Através dos resultados obtidos diante de uma sistemática aferição do que se pretendia, verificou-se que é determinante a dominância das correlações da Imagem Corporal e do Comportamento Alimentar, o progresso da ciência através dos referenciais adotados, desde o inicio comparando e buscando informações pertinentes ao objeto de estudo.

Cabe ressaltar, ainda, que este estudo alerta para a importância que deve ser dada à estudos com estudantes universitárias mediante a inúmeras problemáticas que interferem diretamente no universo das estudantes, a influência sociocultural, a mídia e os apelos das indústrias da moda e empresas de cosméticos que aprisionam primeiramente a consciência, produz uma pseudo imagem do que o ser humano pensa ser, as atribuições intrínsecas evolutivas guardam em sua estrutura a máscara obscura do medo e da imagem de si mesmo, permeando o que na verdade o ser humano deve ser.

Relacionar e avaliar estas variáveis reflete num processo doloroso da concepção daquilo que é belo e desejável, certo de que a contribuição para a ciência está intimamente ligado as concepções daquilo que rejeitamos, uma vez que tratamento e prevenção correlaciona-se a doença, patologia, curas e situações indesejáveis, portanto, doloroso. A satisfação com o corpo tem a ver com mobilidade social, padrão de beleza imposto pela sociedade, bem estar físico e emocional, através de pesquisas podemos desmistificar o 
conceito distorcido daquilo que devemos ser corporalmente, uma vez que se contribuirá diretamente nas concepções do pensamento coletivo social.

\section{Referências}

ALMEIDA, G. A. N. et al. Percepção de tamanho e forma corporal de mulheres: estudo exploratório. Psicologia em Estudo, Maringá, v. 10, n. 1, p. 27-35, 2005.

ALVES, E. et al. Prevalência de sintomas de anorexia nervosa e insatisfação com a imagem corporal em adolescentes do sexo feminino do Município de Florianópolis, Santa Catarina, Brasil. Cadernos de Saúde Pública, Rio de Janeiro, v. 24, n. 3, p. 503-512, 2008.

BALLONE, G. J. Transtornos alimentares em adolescentes. PsiqWeb, 2003. Disponível em: <http://gballone.sites.uol.com.br/alimentar/alimentar2.html>. Acesso em: 20 jan. 2008.

BARROS, D. D. Estudo da imagem corporal da mulher: corpo (ir)real x corpo ideal. 2001. 190 f. Dissertação (Mestrado em Educação Física) - Faculdade de Educação Física, Universidade de Campinas, Campinas, 2001.

CORDÁS, T.; NEVES, J. E. Escalas de avaliação de transtornos alimentares. Revista de Psiquiatria Clínica, São Paulo, v. 26, n. 1, p. 41-47, 1999.

DI PIETRO, M. C. Validade interna, dimensionalidade e desempenho da escala BSQ "Body Shape Questionaire" em uma população de estudantes universitárias. 2001. $88 \mathrm{f}$. Dissertação (Mestrado em Psiquiatria e Psicologia Médica) - Escola Paulista de Medicina, Universidade Federal de São Paulo, São Paulo, 2002.

DURANT, W. The story of philosophy. New York: Washington Square Press, 1961.

GARNER, D. M.; GARFINKEL, P. E. The Eating Attitudes Test: Aan index of the symptoms of anorexia nervosa. Psychological Medicine, London, v. 9, n. 24, p. 273$279,1979$.

GARNER, D. M.; GARFINKEL, P. E.; OLMSTEAD, M. An overview of sociocultural factors in the development of anorexia nervosa. In: DARBY, P. L. et al. (Eds.). Anorexia nervosa: Recent development in research. New York: Alan R. Liss, 1983. p. 65-82.

GIETZEN, D. W. Neural mechanisms in the responses to amino acid deficiency. The Journal of Nutrition, Philadelphia, v. 123, n. 4, p. 610-625, 1993.

KOFES, S. E sobre o corpo, não é o próprio corpo que fala? Ou o discurso desse corpo sobre o qual se fala. In: BRUHNS, H. T. (Org.). Conversando sobre o corpo. Campinas: Papirus, 1985. p. 28-35. 
MORGAN, C. M.; VECCHIATTI, I. R.; NEGRÃO, A. B. Etiologia dos transtornos alimentares: aspectos biológicos, psicológicos e socioculturais. Revista Brasileira de Psiquiatria, São Paulo, v. 3, p. 18-23, 2002. Suplemento 3.

MINTZ, L. B.; O'HALLORAN, M. S. The eating attitudes test: Validation with DSM-IV eating disorder criteria. Journal of Personality Assessment, Burbank, v. 74, n. 3, p. 489-503, 2000.

NAKAMURA, K. et al. Eating problems in female Japonese high school students: A prevalence study. International Journal of Eating Disorders, New York, v. 26, n. 1, p. 91-95, 1999.

NUNES, M. A. et al. Influência da percepção do peso e do índice de massa corporal nos comportamentos alimentares anormais. Revista Brasileira de Psiquiatria, São Paulo, v. 23, n. 1, p. 21-27, 2001.

POKRAJAC-BULIAN, A.; AMBROSI-RANDIC, N. Sociocultural attitudes towards appearance and body dissatisfaction among adolescent girls in Croatia. Eating and Weight Disorders, Milano, v. 12, n. 4, p. e96-e91, 2007.

POKRAJAC-BULIANA, A.; ZIVCIC-BECIREVICACUTE, I.. Locus of control and self-esteem as correlates of body dissatisfaction in Croatian university students. European Eating Disorders Review, Chichester, v. 13, n. 1, p. 54-60, 2005.

RIBEIRO, R. P. P. Indicadores de hábitos dietéticos e aspectos cognitivos e comportamentais relacionados aos distúrbios de conduta alimentar em adolescentes do sexo feminino. 1999. 118 f. Tese (Doutorado em Medicina) - Faculdade de Medicina de Ribeirão Preto, Universidade de São Paulo, Ribeirão Preto, 1999.

RODRÍGUEZ, A. et al. Eating disorders and altered eating behaviors in adolescents of normal weight in a Spanish city. The Journal of Adolescent Health, New York, v. 28, n. 4, p. 338-345, 2001.

STRUBBE, J. H.; GORISSEN, A. Meal patterning in the lactating rat. Physiology $\mathcal{E}$ Behavior, Oxford; v. 25, n. 5, p. 775-777, 1980.

Contato dos autores :

José Carlos de Souza - josecarlossouza@uol.com.br

Texto Recebido em: 10 /02/2017

Aceito em: 23 /09/2017 